\title{
ABOUT THE PROJECT ÜBER DAS PROJEKT
}

This book is a documentation of research work as well as a negotiation of concepts and ideas that emerged from within Conceptual Joining, a project at the University of Applied Arts Vienna, funded by the Austrian Science Fund (FWF) within the Programme for Arts-based Research (PEEK).

The team investigated the specific characteristics of wood as well as historic, existing and potential processing techniques for that material with the aim of deriving guiding principles for the design and materialization of architectural scenarios. This process was supported by international collaboration partners and experts from various disciplines.
Das vorliegende Buch ist eine Dokumentation der Forschungsarbeit sowie eine Verhandlung von Konzepten und Ideen, die im Rahmen des Forschungsprojekts Conceptual Joining entstanden sind. Das Projekt an der Universität für angewandte Kunst Wien wird vom Österreichischen Wissenschaftsfonds (FWF) im Rahmen des Programms zur Entwicklung und Erschließung der Künste (PEEK) gefördert.

Ein Team von Forscher*innen untersuchte die spezifischen Eigenschaften von Holz sowie bestehende und mögliche Verarbeitungstechniken mit dem Ziel, Leitprinzipien für den Entwurf und die Materialisierung von architektonischen Szenarien abzuleiten. Unterstützt wurde dieser Prozess durch internationale Kooperationspartner*innen und Expert*innen aus unterschiedlichen Disziplinen.

Project Lead / Projektleitung:

Christoph Kaltenbrunner

Mentors / Mentor*innen:

Anja Jonkhans, Clemens Preisinger, Karin Raith

Researchers / Forscher*innen:

Lukas Allner, Daniela Kröhnert, Philipp Reinsberg, Mechthild Weber

In collaboration with / Zusammenarbeit mit:

Florian Fend, Philipp Hornung, Ben James, Kanade Nagata, Adam Orlinski, Andrea Rossi, Jun Sato, Shigeatsu Shimizu, Matthew Tam, Alfred Teischinger, Sandra Tuider, Landesberufsschule Murau (Vocational School Murau) 